\title{
Growth inhibition and induction of apoptosis in SGC-7901 human gastric cancer cells by evodiamine
}

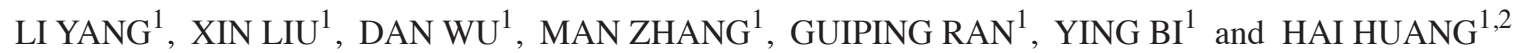 \\ ${ }^{1}$ School of Clinical Laboratory Science, Guiyang Medical University; ${ }^{2}$ Department of Clinical Biochemistry, \\ Affiliated Hospital of Guiyang Medical University, Guiyang, Guizhou 550004, P.R. China
}

Received May 12, 2013; Accepted January 10, 2014

DOI: $10.3892 / \mathrm{mmr} .2014 .1924$

\begin{abstract}
Evodiamine is one of the major bioactive compounds isolated and purified from the fruit of Fructus Evodiae. Numerous studies have indicated that evodiamine exhibits activity against human tumor cells. In the present study, the effect of evodiamine on the proliferation and apoptosis of SGC-7901 human gastric cancer cells and the correlative mechanisms were investigated. This may provide further experimental evidence of the pharmacological actions of evodiamine and a strategy for its use as a novel chemotherapeutic drug. Following treatment with evodiamine, the typical morphological changes of apoptosis were observed in human SGC-7901 cells. Cell cycle analysis indicated that evodiamine induced G2/M phase arrest in SGC-7901 cells and flow cytometry revealed that evodiamine induced apoptosis. Analysis of the enzymatic activity demonstrated that evodiamine increased the activity of caspase-3, -8 and -9 in SGC-7901 cells. The protein expression of caspase- $3,-8$ and -9 and Bax increased, and the expression of Bcl-2 decreased following treatment with evodiamine. These results suggest that evodiamine is able to inhibit the proliferation of SGC-7901 cells by inhibiting the cell cycle at G2/M phase and inducing apoptosis in SGC-7901 cells by activating caspase- $-3,-8$ and -9 , and altering the expression of caspase-3, Bax and Bcl-2.
\end{abstract}

\section{Introduction}

Fructus Evodiae, a traditional Chinese medicine, has been used in the treatment of headache, abdominal pain, postpartum hemorrhage, dysentery and amenorrhea (1). Evodiamine is the most important quinoline alkaloid isolated from the fruit of Fructus Evodiae. Evodiamine has been demonstrated to possess numerous biological effects, including analgesic,

Correspondence to: Dr Hai Huang, Department of Clinical Biochemistry, Guiyang Medical University, No. 9 Beijing Road, Guiyang, Guizhou 550004, P.R. China

E-mail: huanghai828@gmc.edu.cn

Key words: evodiamine, SGC-7901 cells, growth inhibition, apoptosis, cell cycle anti-inflammatory, antiobesity, vasodilatory, thermoregulatory and uterotonic effects (1). In recent years, studies investigating the anticancer mechanisms of evodiamine have become a major topic of focus. Certain studies have suggested that evodiamine has anticancer activity by inducing apoptosis, arresting the cell cycle and inhibiting tumor invasion and metastasis. Evodiamine has been demonstrated to inhibit the proliferation of multiple tumor cells, including human leukemia (2), malignant melanoma (3-5), thyroid carcinoma (6), prostate cancer (7-9), breast cancer $(10)$, hepatoma $(11,12)$, cervical cancer $(5,6,13)$, colon carcinoma $(14,15)$ and pancreatic cancer $(16)$ cells.

Cancer is not only a disease exhibiting abnormal cellular proliferation and differentiation, but is also a disease with abnormal apoptosis. Previous studies on apoptosis and its mechanisms have provided a novel method for overcoming cancer, that is, the induction of apoptosis in tumor cells rather than the inhibition of tumor cell proliferation. Various methods may be used to induce apoptosis in tumor cells or to establish the optimal conditions for this induction.

Gastric carcinoma is one of the most common types of malignancy and is the leading cause of cancer-related mortality in China (17). Chemotherapy is one of the main therapeutic approaches for treating gastric cancer. However, the effect of evodiamine on gastric carcinoma remains poorly defined and the exact mechanisms are unclear. Examining the molecular mechanisms underlying the effects of evodiamine on gastric carcinoma cells may provide novel methods for the treatment of gastric cancer and add to the growing evidence that evodiamine may be used as a systemic anticancer drug.

The SGC-7901 human gastric cancer cell line is an ideal cellular model to study the proliferation and differentiation of gastric cancer in vitro, which has the advantages of a simple in vitro amplification and rapid entry to the exponential phase. Consequently, the present study selected the SGC-7901 cell line for investigation.

Our previous study (18) demonstrated that evodiamine inhibits the proliferation and induces apoptosis of SGC-7901 gastric cancer cells, and the half effective inhibitory concentration $\left(\mathrm{IC}_{50}\right)$ of evodiamine is $1.5 \mu \mathrm{mol} / \mathrm{l}$. In the present study, SGC-7901 cells were treated with $1.5 \mu \mathrm{mol} / 1$ evodiamine for different time periods and the effects on proliferation and apoptosis were observed. The present study investigates the possible molecular mechanisms by which evodiamine affects SGC-7901 cells. 


\section{Materials and methods}

Materials. Evodiamine was obtained from the National Institutes for Food and Drug Control (Beijing, China). RPMI-1640 medium, penicillin-streptomycin and $0.25 \%$ trypsin solution were purchased from Hyclone (Logan, UT, USA). L-Glutamine, Hanks' balanced salt solution and dimethylsulfoxide (DMSO) were obtained from Solabio (Beijing, China). Fetal bovine serum (FBS) was purchased from Hangzhou Sijiqing Biological Engineering Materials Co., Ltd. (Hangzhou, China). The Hoechst staining kit, Bradford protein assay kit, caspase-3 activity assay kit, caspase- 8 activity assay kit, caspase- 9 activity assay kit, enhanced bicinchoninic acid (BCA) protein assay kit and RIPA lysis buffer were purchased from the Beyotime Institute of Biotechnology (Shanghai, China). The Annexin V-fluorescein isothiocyanate apoptosis detection kit and cell cycle detection kit were purchased from KeyGen Biotech Co., Ltd. (Nanjing, China). Caspase-3, Bax, Bcl-2, $\beta$-actin, horseradish peroxidase (HRP)-conjugated goat anti-mouse IgG and HRP-conjugated goat anti-rabbit IgG antibodies were obtained from Santa Cruz Biotechnology, Inc. (Santa Cruz, CA, USA).

Cell culture and morphological analysis. The SGC-7901 human gastric cancer cell line was obtained from the Shanghai Type Culture Collection of the Chinese Academy of Sciences (Shanghai, China). The cells were cultured in RPMI-1640 medium supplemented with $10 \% \mathrm{FBS}, 100 \mathrm{U} / \mathrm{ml}$ penicillin and $100 \mathrm{mg} / \mathrm{l}$ streptomycin, at $37^{\circ} \mathrm{C}$ in a humidified atmosphere of $95 \%$ air and $5 \% \mathrm{CO}_{2}$. Following $48 \mathrm{~h}$, the medium was removed and replaced by medium containing $1.5 \mu \mathrm{mol} / \mathrm{l}$ evodiamine or a drug-free medium (control condition) for 12, 24 and $36 \mathrm{~h}$. The morphology of SGC-7901 cells was monitored under an inverted contrast phase microscope (Nikon TE 2000-U; Nikon Tokyo, Japan) at 12, 24 and $36 \mathrm{~h}$.

Hoechst 33258 staining. The SGC-7901 cells seeded on the coverslips were treated with $1.5 \mu \mathrm{mol} / \mathrm{l}$ evodiamine for the indicated time points and the coverslips were then collected. Hoechst 33258 staining was performed on the coverslips to observe the morphological cell changes. Attached cells were washed twice with phosphate-buffered saline (PBS) and fixed with fixation fluid (paraformaldehyde) for $10 \mathrm{~min}$. The fixation fluid was then removed and the cells were washed twice with PBS prior to staining with $500 \mu$ l Hoechst 33258. Following staining for $5 \mathrm{~min}$, the cells were washed twice again and observed under a fluorescence microscope (Nikon TE 2000-U) with ultraviolet light.

Flow cytometric cell cycle analysis. SGC-7901 cells were incubated in six-well plates. Following treatment with $1.5 \mu \mathrm{mol} / 1$ of evodiamine for 12,24 and $36 \mathrm{~h}$, the cells were harvested and washed with PBS. The pelleted cells were fixed in ice-cold $70 \%$ ethanol at $4^{\circ} \mathrm{C}$ overnight. The fixed cells were washed twice with PBS and the cells were treated with $100 \mu \mathrm{l}$ RNase stock solution and incubated at $37^{\circ} \mathrm{C}$ for $30 \mathrm{~min}$. The cells were stained with $400 \mu \mathrm{l}$ propidium iodide staining solution at $4^{\circ} \mathrm{C}$ for $30 \mathrm{~min}$ in the dark. The stained cells were analyzed by flow cytometry based on red fluorescence.
Caspase activity assay. SGC-7901 cells treated with evodiamine were harvested and washed with ice-chilled PBS. The cell pellets were resuspended with an appropriate quantity of lysis buffer (Tris- $\mathrm{HCl}$ ) for $15 \mathrm{~min}$ in an ice bath. Following centrifugation $\left(16,000 \mathrm{xg}\right.$ at $\left.4^{\circ} \mathrm{C}\right)$ for $15 \mathrm{~min}$, the supernatant was transferred into ice-chilled centrifuge tubes. The protein concentration was measured by the Bradford assay. Then the enzyme activity of caspase- $3,-8$ and -9 was detected according to the manufacturer's instructions of the caspase activity assay kit (Beyotime Institute of Biotechnology).

Western blot analysis. SGC-7901 cells were cultured in RPMI-1640 medium until mid-log phase and then incubated with $1.5 \mu \mathrm{mol} / 1$ evodiamine for 12,24 and $36 \mathrm{~h}$. The cells were harvested and the proteins were isolated using RIPA lysis buffer (Beyotime Institute of Biotechnology). Total protein was measured using the enhanced BCA protein assay kit (Beyotime Institute of Biotechnology). Equal quantities of protein samples were separated by SDS-PAGE and transferred onto the polyvinylidene fluoride membranes (Millipore, Billerica, MA, USA). Western blotting was performed using antibodies against caspase-3, Bax and Bcl-2 (Santa Cruz Biotechnology, Inc.). The secondary antibodies used were goat anti-mouse and goat anti-rabbit (Santa Cruz Biotechnology, Inc.) HRP-labeled antibodies. The signals were visualized by enhanced chemiluminescence detection (Millipore).

Statistical analysis. All experiments were repeated three times and all values are expressed as the mean \pm standard deviation. The independent-samples t-test was calculated to compare the mean of each group with that of the control group. $\mathrm{P}<0.05$ was considered to indicate a statistically significant difference.

\section{Results}

Effect of evodiamine on cellular morphology. Following treatment with evodiamine, the morphological changes in the cells were observed by inverted microscopy. In the evodiamine groups, SGC-7901 cells became irregular and exhibited shrinkage. Detachment of the cells from the cell culture substratum was observed (Fig. 1). These changes were characteristic of apoptotic cell death. In the control groups, cell morphology did not change significantly.

Hoechst 33258 staining was used to detect chromatin condensation, one of the typical morphological features of apoptosis. The Hoechst 33258 dye stained morphologically normal nuclei dimly blue, whereas evodiamine-treated cells demonstrated smaller nuclei with brilliant blue staining (Fig. 2). Compared with the control cells, the cells exposed to evodiamine presented typical apoptotic morphology. These results demonstrate that evodiamine induces the morphological changes of apoptotic cell death in SGC-7901 cells.

Effects of evodiamine on cell cycle progression. To investigate whether the antiproliferative effect of evodiamine was associated with cell cycle arrest, analysis of cell cycle phase distribution was performed following treatment with evodiamine using flow cytometry. Evodiamine treatment resulted in the accumulation of SGC-7901 cells at the G2/M phase (Fig. 3A). Following treatment with evodiamine, the number of 


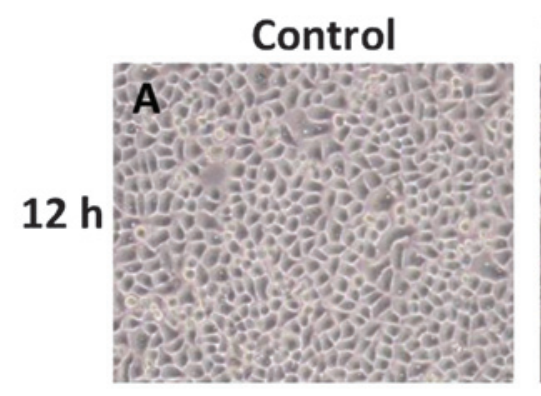

\section{$1.5 \mu \mathrm{M}$ Evodiamine}
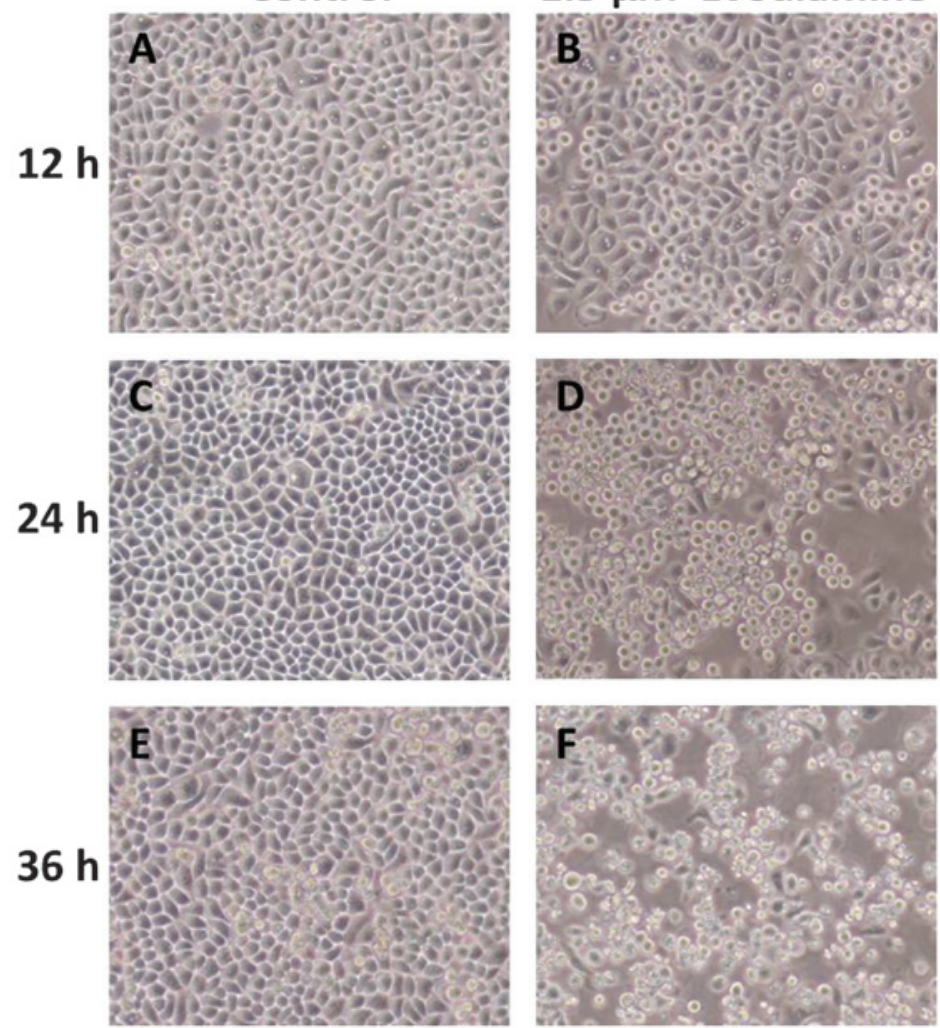

Figure 1. Morphological alterations of SGC-7901 cells observed by inverted microscopy. (A) Normal control group at 12 h; (B) evodiamine group at $12 \mathrm{~h}$; (C) normal control group at $24 \mathrm{~h}$; (D) evodiamine group at $24 \mathrm{~h}$; (E) normal control group at $36 \mathrm{~h}$; and (F) evodiamine group at $36 \mathrm{~h}$. Magnification, x100.

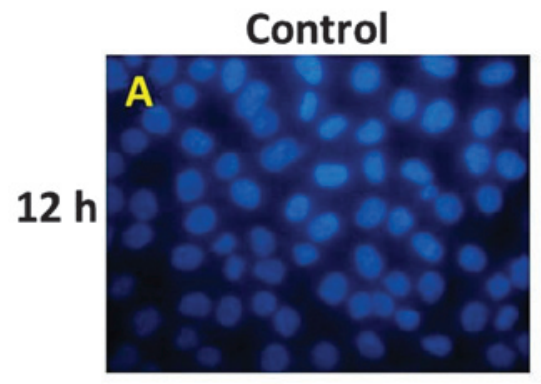

\section{$1.5 \mu \mathrm{M}$ Evodiamine}
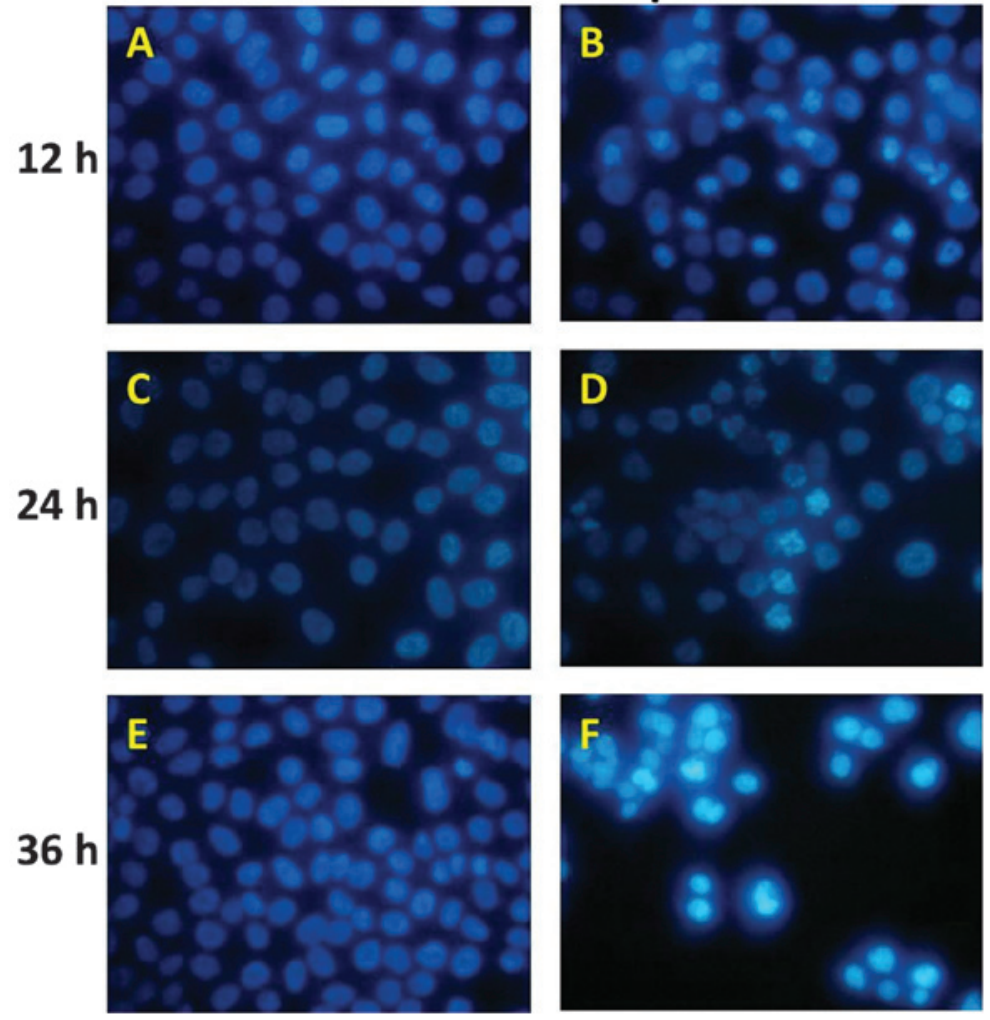

Figure 2. Morphological alterations of cells stained with Hoechst 33258 were observed by fluorescent microscopy. (A) Normal control group at $12 \mathrm{~h}$; (B) evodiamine group at $12 \mathrm{~h}$; (C) normal control group at $24 \mathrm{~h}$; (D) evodiamine group at $24 \mathrm{~h}$; (E) normal control group at $36 \mathrm{~h}$; and (F) evodiamine group at $36 \mathrm{~h}$. Compared with the control cells, the cells exposed to evodiamine presented typical apoptotic morphology. Hoechst 33258 dye stained morphologically normal nuclei dimly blue, whereas evodiamine-treated cells demonstrated smaller nuclei with brilliant blue staining. Magnification, x200. 
A

\section{Control}
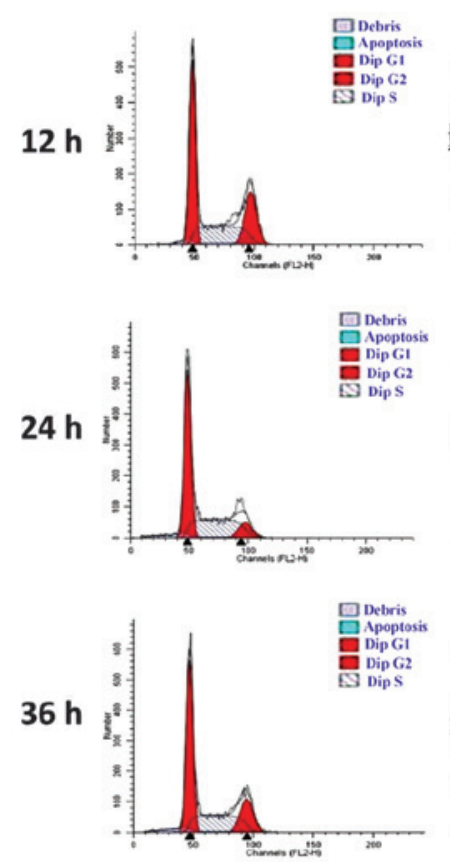

\section{$1.5 \mu \mathrm{M}$ Evodiamine}
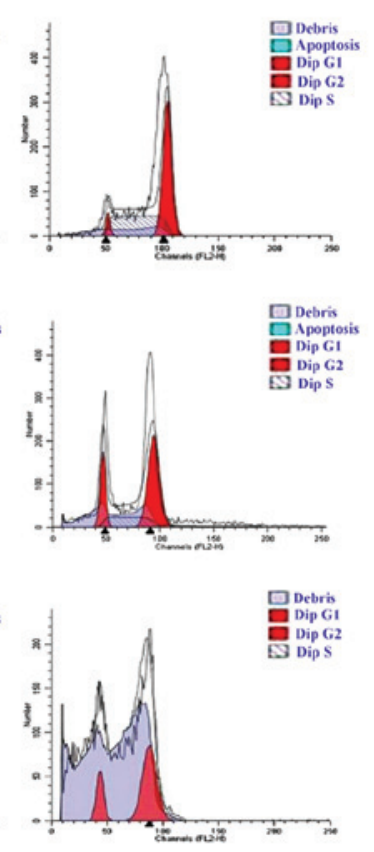

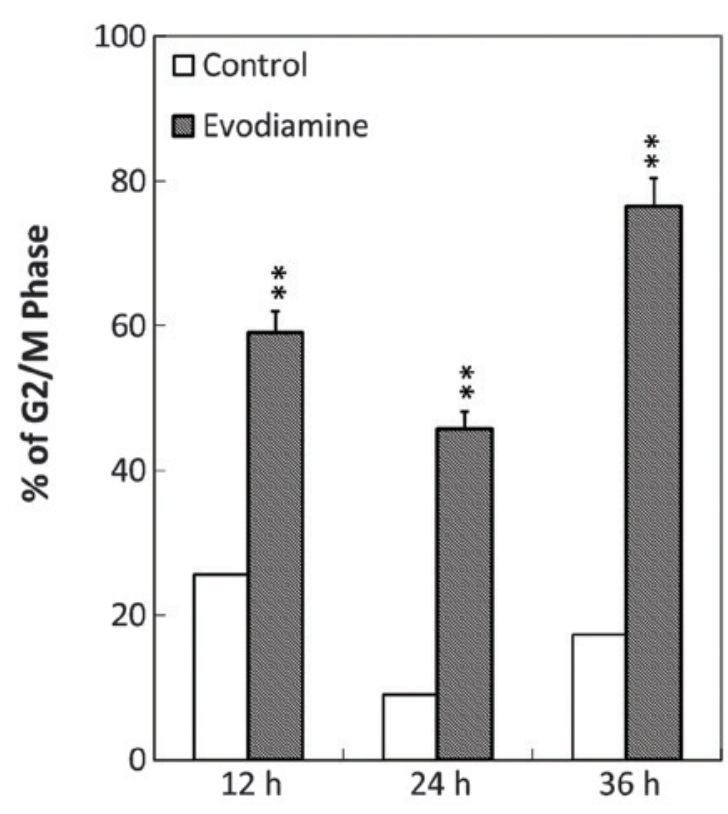

Figure 3. Detection of evodiamine-induced cell cycle distribution in SGC-7901 cells. (A) Histograms depicting cell cycle distribution in SGC-7901 cells treated with $1.5 \mu \mathrm{M}$ evodiamine. (B) Bar graph of the G2/M phase of SGC-7901 cells treated with $1.5 \mu \mathrm{M}$ evodiamine. The results are expressed as the means \pm standard error of the mean of three independent experiments. ${ }^{* *} \mathrm{P}<0.01$ versus control.
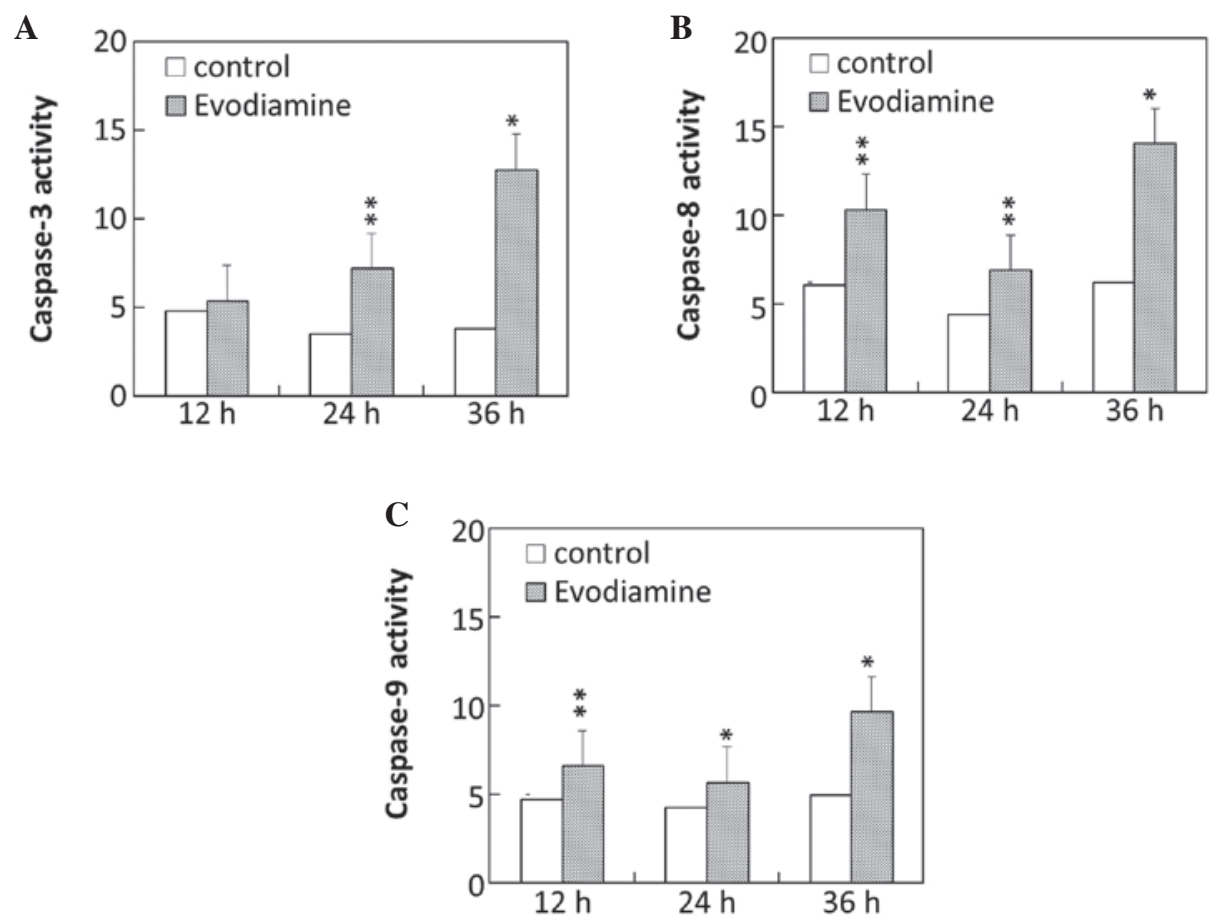

Figure 4. Activity changes of caspase-3, -8 and -9 in SGC-7901 cells treated with $1.5 \mu \mathrm{M}$ evodiamine. The results are expressed as the means \pm standard error of the mean of three independent experiments. ${ }^{*} \mathrm{P}<0.05$ and ${ }^{* *} \mathrm{P}<0.01$ versus control.

SGC-7901 cells that arrested at the G2/M phase was elevated by $34.4,44.4$ and $59.2 \%$ at 12,24 and $36 \mathrm{~h}$, respectively (Fig. 3B). These results indicated that evodiamine was able to affect the distribution of the cell cycle and induce $\mathrm{G} 2 / \mathrm{M}$ arrest in SGC-7901 cells.
Effects of evodiamine on caspase activity. To investigate the molecular mechanism of evodiamine-induced apoptosis, the activity of caspase-3, -8 and -9 in SGC-7901 cells was examined. The results demonstrated that the caspase-3 activity of SGC-7901 cells was markedly increased following treatment 


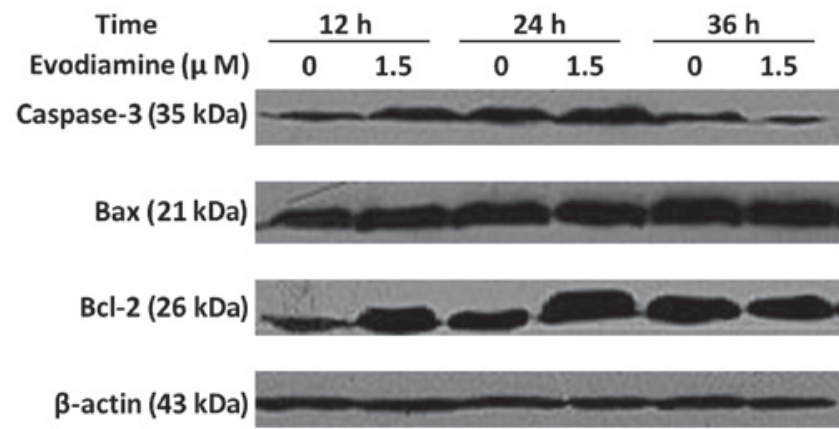

Figure 5. Expression of apoptosis-related proteins in SGC-7901 cells. Cells were incubated with evodiamine. Cell lysates were extracted and analyzed by western blotting. Similar results were obtained in three separate experiments.

with evodiamine for 24 and 36 h (Fig. 4A). Evodiamine increased the activity of caspase-8 in SGC-7901 cells following exposure for 12, 24 and $36 \mathrm{~h}$ (Fig. 4B). The activity of caspase-9 was elevated following treatment with evodiamine for 12, 24 and $36 \mathrm{~h}$ (Fig. 4C).

Western blot analysis of caspase-3, Bax and Bcl-2 protein. The treatment of SGC-7901 cells with $1.5 \mu \mathrm{mol} / 1$ evodiamine for $24 \mathrm{~h}$ resulted in an increase in caspase-3 expression (Fig. 5). Bax expression was elevated following incubation with evodiamine for 24 and $36 \mathrm{~h}$. The expression of Bcl-2 was increased following evodiamine treatment for 12 and $24 \mathrm{~h}$ and decreased following $36 \mathrm{~h}$. The expression of $\beta$-actin served as an internal control. These data demonstrated that evodiamine induced the apoptosis of SGC-7901 cells through a caspase-dependent pathway.

\section{Discussion}

Apoptosis is an autonomic ordered, programmed, physiological mode of cell death that is important in tissue homeostasis and is controlled by serial genes. Cancer is not only a disease with abnormal cellular proliferation and differentiation but also a disease with abnormal apoptosis. Numerous types of tumors are the result of an imbalance between cell proliferation and cell death. Thus, investigating the basic mechanisms of apoptosis may provide insight into potential novel therapeutic targets and have vital significance.

Evodiamine is one of the major bioactive compounds isolated and purified from the fruit Fructus Evodiae. Several studies $(2,6-8,10)$ have indicated that evodiamine exhibits activity against human tumor cells. Our previous study (18) demonstrated that evodiamine inhibits the proliferation of SGC-7901 gastric cancer cells. In the present study, the molecular mechanisms by which evodiamine affects SGC-7901 cells were investigated and novel evidence was identified for the future medical use of evodiamine. The previous study determined whether evodiamine was toxic for normal peripheral blood mononuclear cells (PBMCs). The results demonstrated that evodiamine exhibited less toxicity in PBMCs that were treated with various concentrations of evodiamine for $24 \mathrm{~h}$. From the early tests it was concluded that evodiamine-induced cell death was mediated by time- and dose-dependent pathways, and the optimal concentration and exposure time was $1.5 \mu \mathrm{m} / 1$ for $24 \mathrm{~h}$. The present study also demonstrated that DMSO, as a co-solvent, exhibited no cytotoxic effect on SGC-7901 cells. Therefore, the $\mathrm{IC}_{50}$ of evodiamine was selected to act upon SGC-7901 cells.

Morphological alteration is an important characteristic of apoptosis. Alterations, including cell shrinkage, cell detachment, chromatin condensation, nuclear fragmentation and formation of apoptotic bodies are characteristic of cell apoptotic death. Cellular morphological analysis demonstrated that SGC-7901 cells underwent typical apoptotic changes following treatment with evodiamine.

Evodiamine has been demonstrated to induce cell cycle arrest in prostate $(7,8)$, breast $(10)$ and colorectal cancer $(14,15)$. Flow cytometry indicated that evodiamine induced cell cycle arrest at the G2/M phase in SGC-7901 cells. The cells in G2/M phase mainly synthesize RNA and protein, and prepare for cell division. The present study inferred that evodiamine may affect the synthesis of proteins that are essential for cells, thus inhibiting the proliferation of SGC-7901 cells.

In order to gain insight into the molecular mechanisms underlying the effects of evodiamine in apoptosis, the enzyme activity of caspase-3, -8 and -9 was determined. Numerous internal and external signal stimuli are able to induce apoptosis. Although there are various signals and pathways that follow, it is generally considered that the common pathway of late apoptosis is the activation of the caspase family. Caspases are the central executioners in the apoptotic process. Caspases are divided into two functional subfamilies, including promoter caspases (caspase-8 and -9) and effector caspases (caspase-3 and -7). The activated promoter caspase is able to activate effector caspases, thus completing apoptosis. Data from the present study demonstrated that caspase-3, - 8 and -9 were activated by evodiamine following incubation for 24 and $36 \mathrm{~h}$, indicating that evodiamine resulted in apoptosis of SGC-7901 cells mediated by the activation of caspase-3, - 8 and -9 . Furthermore, western blotting demonstrated that the expression of caspase- 3 also increased following evodiamine treatment for 24 and $36 \mathrm{~h}$.

The Bcl-2 protein family and its members form a complicated interactive network, which is able to regulate apoptosis. Among these proteins, the expression of Bax and $\mathrm{Bcl}-2$ is directly associated with apoptosis regulation. The increased expression of Bax and decreased expression of $\mathrm{Bcl}-2$ is able to promote apoptosis. In the present study, data suggested that the expression of Bax was increased following evodiamine treatment for 24 and 36 h. Evodiamine increased Bcl-2 expression at 12 and $24 \mathrm{~h}$ and decreased Bcl-2 expression at $36 \mathrm{~h}$. Bcl-2 is important in tumorigenesis by inhibiting the signals that induce apoptosis. Bcl-2 is also phosphorylated in vivo and this modification has been demonstrated to effect its anti-apoptotic activity (19). In addition, Bcl-2 is able to inhibit or delay apoptosis induced by various stimuli, including chemotherapeutic drugs. As a result, the present study inferred that, in the early stages of evodiamine action, increased $\mathrm{Bcl}-2$ expression may be a delayed action of cells on external stimulated factors, so as to prevent the cytotoxic effect of evodiamine and protect the cells. However, it was not able to prevent apoptosis and the rate of apoptosis at this stage was elevated. When drug treatment was prolonged to $36 \mathrm{~h}$, the decreased Bcl-2 expression may have been associated with the stimulatory effect of 
evodiamine exceeding the cellular regulatory activity, which may have been responsible for the induction of apoptosis in SGC-7901 cells. The results indicate that the apoptosis caused by evodiamine in SGC-7901 cells may be associated with the alteration of the $\mathrm{Bcl}-2$ protein family.

In conclusion, the results of the present study suggest that evodiamine inhibits the proliferation of SGC-7901 cells by arrest at the $\mathrm{G} 2 / \mathrm{M}$ phase. In addition, evodiamine is able to activate caspase- $3,-8$ and -9 , upregulate the expression of caspase- 3 and Bax, and downregulate the expression of Bcl-2, potentially the mechanism by which evodiamine induces the apoptosis of SGC-7901 cells.

\section{Acknowledgements}

This study was supported by a research grant from the National Natural Science Foundation of China (81260658).

\section{References}

1. Jiang J and Hu C: Evodiamine: a novel anti-cancer alkaloid from Evodia rutaecarpa. Molecules 14: 1852-1859, 2009.

2. Lee TJ, Kim EJ, Kim S, Jung EM, Park JW, Jeong SH, Park SE, Yoo YH and Kwon TK: Caspase-dependent and caspase-independent apoptosis induced by evodiamine in human leukemic U937 cells. Mol Cancer Ther 5: 2398-2407, 2006.

3. Zhang Y, Wu LJ, Tashiro S, Onodera S and Ikejima T: Intracellular regulation of evodiamine-induced A375-S2 cell death. Biol Pharm Bull 26: 1543-1547, 2003.

4. Wang C, Wang MW, Tashiro S, Onodera S and Ikejima T: Roles of SIRT1 and phosphoinositide 3-OH kinase/protein kinase C pathways in evodiamine-induced human melanoma A375-S2 cell death. J Pharmacol Sci 97: 494-500, 2005.

5. Zhang Y, Wu LJ, Tashiro S, Onodera S and Ikejima T: Evodiamine induces tumor cell death through different pathways: apoptosis and necrosis. Acta Pharmacol Sin 25: 83-89, 2004

6. Chen MC, Yu CH, Wang SW, Pu HF, Kan SF, Lin LC, Chi CW, Ho LL, Lee CH and Wang PS: Anti-proliferative effects of evodiamine on human thyroid cancer cell line ARO. J Cell Biochem 110: 1495-1503, 2010.
7. Kan SF, Huang WJ, Lin LC and Wang PS: Inhibitory effects of evodiamine on the growth of human prostate cancer cell line LNCaP. Int J Cancer 110: 641-651, 2004.

8. Kan SF, Yu CH, Pu HF, Hsu JM, Chen MJ and Wang PS: Anti-proliferative effects of evodiamine on human prostate cancer cell lines DU145 and PC3. J Cell Biochem 101: 44-56, 2007.

9. Huang DM, Guh JH, Huang YT, Chueh SC, Chiang PC and Teng CM: Induction of mitotic arrest and apoptosis in human prostate cancer pc-3 cells by evodiamine. J Urol 173: 256-261, 2005.

10. Liao CH, Pan SL, Guh JH, Chang YL, Pai HC, Lin CH and Teng CM: Antitumor mechanism of evodiamine, a constituent from Chinese herb Evodiae fructus, in human multiple-drug resistant breast cancer NCI/ADR-RES cells in vitro and in vivo. Carcinogenesis 26: 968-975, 2005.

11. Wang XN, Han X, Xu LN, Yin LH, Xu YW, Qi Y and Peng JY: Enhancement of apoptosis of human hepatocellular carcinoma SMMC-7721 cells through synergy of berberine and evodiamine. Phytomedicine 15: 1062-1068, 2008.

12. Zhu LH, Liu XD, Tan YH, Li JF, Du BY and Wu YY: Proliferation-inhibited and apoptosis-inducted effects of evodiamine on human hepatoma cell line HepG2. Chinese Pharmacological Bulletin 1: 68-71, 2009.

13. Fei XF, Wang BX, Li TJ, Tashiro S, Minami M, Xing DJ and Ikejima T: Evodiamine, a constituent of Evodiae Fructus, induces anti-proliferating effects in tumor cells. Cancer Sci 94: 92-98, 2003.

14. Ogasawara M, Matsubara T and Suzuki H: Inhibitory effects of evodiamine on in vitro invasion and experimental lung metastasis of murine colon cancer cells. Biol Pharm Bull 24: 917-920, 2001.

15. Ogasawara M and Suzuki H: Inhibition by evodiamine of hepatocyte growth factor-induced invasion and migration of tumor cells. Biol Pharm Bull 27: 578-582, 2004

16. Wei WT, Chen H, Wang ZH, Ni ZL, Liu HB, Tong HF, Guo HC, Liu DL and Lin SZ: Enhanced antitumor efficacy of gemcitabine by evodiamine on pancreatic cancer via regulating PI3K/Akt pathway. Int J Biol Sci 8: 1-14, 2012.

17. Chen W, Zheng R, Zhang S, et al: The incidences and mortalities of major cancers in China, 2009. Chin J Cancer 32: 106-112, 2013.

18. Huang H, Zhang Y, Liu X, Li Z, Xu W,He S, Huang Y and Zhang H: Acid sphingomyelinase contributes to evodiamine-induced apoptosis in human gastric cancer SGC-7901 cells. DNA Cell Biol 30: 407-412, 2011.

19. Gross A, McDonnell JM and Korsmeyer SJ: BCL-2 family members and the mitochondria in apoptosis. Genes Dev 13: 1899-1911, 1999. 